\title{
Hyperglycaemia is associated with cancer-related but not non-cancer-related deaths: evidence from the IPC cohort
}

\author{
Jean-Marc Simon ${ }^{1,2} \cdot$ Frederique Thomas $^{1}$ - Sebastien Czernichow ${ }^{3,4}$. Olivier Hanon ${ }^{1,5} \cdot$ Cedric Lemogne $^{3,4,6}$. \\ Tabassome Simon ${ }^{7}$ • Bruno Pannier ${ }^{1,8}$ • Nicolas Danchin ${ }^{1,3,9}$
}

Received: 13 September 2017 / Accepted: 1 December 2017 / Published online: 5 January 2018

(C) Springer-Verlag GmbH Germany, part of Springer Nature 2018

\begin{abstract}
Aims/hypothesis Hyperglycaemia has been associated with the incidence of all and specific types of cancer, distinct from the risks related to diabetes. The relationships between blood glucose and mortality rates related to all and specific cancers were analysed in comparison with all-cause or non-cancer-related mortality rates in a large, general primary care population in France. Methods Between January 1991 and December 2008, 301,948 participants (193,221 men and 108,727 women), aged 1695 years (mean \pm SD $44.8 \pm 12.0$ years for men and $45.1 \pm 14.2$ years for women), had a health check at the IPC Centre. All data collected in standard conditions during the health checks-up were used for statistical analysis All examinations were performed under fasting conditions and included a blood glucose measurement. Participants with known diabetes $(<9 \%)$ were excluded from the analysis. Participants were classified into quintiles based on their blood glucose measurement and were followed for a maximum of 17 years (mean $\pm \mathrm{SD} 9.2 \pm 4.7$ years) to assess all-cause, cancer and non-cancer mortality rates. Results A non-linear relationship was observed between cancer mortality rates and blood glucose quintile after adjustment for age and sex. There was a significant association between the group with the highest blood glucose level and cancer-related death (multivariate Cox model, HR [95\% CI] 1.17 [1.03, 1.34]), while the group with normoglycaemia showed no association with cancer-related deaths. We did not observe a relationship between blood glucose and all-cause or non-cancer mortality rates. An excess risk of death was observed in the highest blood glucose quintile for gastrointestinal cancer and leukaemia. Adjustments for diabetes and aspirin use did not modify the results. However, this excess risk disappeared with use of glucose-lowering agents (HR [95\% CI] $1.03[0.74,1.43])$.

Conclusions/interpretation Hyperglycaemia is associated with significantly higher rates of cancer-related deaths, particularly in gastrointestinal cancer and leukaemia, but not with non-cancer-related deaths. The association is retained when taking into account confounding factors, including chronic aspirin treatment.
\end{abstract}

Keywords Cancer $\cdot$ Epidemiology $\cdot$ Hyperglycaemia $\cdot$ Mortality

\section{Abbreviations \\ AMORIS Apolipoprotein MOrtality RISk \\ IPC Centre Centre d'Investigations Préventives et Cliniques}

Bruno Pannier pannier@ipc.asso.fr

Centre IPC, 6-14 rue La Pérouse, 75016 Paris, France

Hopital Pitié Salpétrière, APHP, Paris, France

3 Hopital HEGP, APHP, Paris, France

4 Hôpitaux Universitaires Paris Ouest, APHP, Paris, France

\section{Introduction}

A mutual association between diabetes and cancer, two very common diseases worldwide, has been suggested by epidemiological surveys in various populations, and confirmed in

5 Hopital Broca, APHP, Paris, France

6 Inserm U894, Centre Psychiatrie et Neurosciences, Paris, France

7 Hopital Saint Antoine, APHP, Université Pierre et Marie Curie, Paris, France

8 Hopital Manhès, Fleury-Merogis, France

9 Université Paris Descartes, Sorbonne Paris Cité, Faculté de médecine, Paris, France 


\section{Research in context}

\section{What is already known about this subject?}

- Significant relationships have been observed between cancer and type 2 diabetes

- Recently, hyperglycaemia, rather than diabetes, has been implicated in these relationships

\section{What is the key question?}

- Does a relationship exist between hyperglycaemia and cancer- and non-cancer-related mortality rates?

\section{What are the new findings?}

- Hyperglycaemia was associated with an increased risk of cancer-related death

- The group with the highest blood glucose levels had a significantly increased risk of cancer-related death but not of non-cancer-related death. This difference was maintained following adjustment for confounding factors, specifically the use of various medications, including aspirin

- An excess risk of death was observed in the highest blood glucose quintile for gastrointestinal cancer and leukaemia

- We observed an additional benefit of glucose-lowering medications in terms of a reduction in cancer-related deaths

How might this impact on clinical practice in the foreseeable future?

- Common risk factors or the inflammation associated with glucose metabolism may play a deleterious role in the evolution of cancer

an expert consensus statement [1]. Although small, this association was nonetheless found significant in a large metaanalysis and a survey on the prevalence of all cancer types in individuals with type 2 diabetes [2, 3]. Experts suggest that this link may be explained by common risk factors and/or mechanistic relationships, such as inflammation. However, while colorectal cancer mortality rates were increased in individuals with diabetes, this relationship was primarily explained by the cardiovascular complications of diabetes and not by any increase in cancer-specific mortality [4]. Furthermore, it would appear that prostate cancer has a low prevalence in individuals with diabetes relative to other cancers (i.e. liver, pancreas, endometrium, colon/rectum, breast and bladder). Thus, the relationship between diabetes and cancer is yet to be definitively ascertained. Two recent studies have suggested a direct involvement of hyperglycaemia in the glucose metabolism-cancer relationship [5, 6].

An impact of glucose-lowering drugs on cancer incidence has also been postulated. First, the use of metformin, a firstline treatment for diabetes worldwide, has been shown to be inversely associated with cancer incidence. A meta-analysis of mainly retrospective studies that compared metformin with other glucose-lowering treatments, including insulin, revealed a reduction in cancer incidence, with the exception of colon and breast cancer, in individuals with diabetes [7]. In contrast, insulin therapy, both generic and specific compounds [8], has been linked with an increased risk of pancreatic cancer; however, a post hoc analysis using glargine clearly showed no association with cancer incidence [9].
Several meta-analyses have suggested that aspirin may reduce the incidence of different types of cancer or the incidence of metastasis [10, 11]. Recent guidelines from the US Preventive Services Task Force state that aspirin use in 50-69year-old individuals with high cardiovascular risk has a low but potentially interesting secondary benefit in preventing colorectal cancer over several decades [12]. Given that antiaggregant therapy with aspirin is very common in individuals with diabetes, testing for such an interaction may prove valuable in the study of the relationship between hyperglycaemia and cancer incidence.

The aim of the present study was to assess the relationships between fasting hyperglycaemia and cancer- or non-cancerrelated death in a large French cohort of primary care volunteers. We also analysed the mortality rates associated with specific cancer types, and the influence of glucose-lowering agents and regular aspirin treatment on mortality rates.

\section{Methods}

Participants Volunteers were recruited at the Centre d'Investigations Préventives et Cliniques (IPC Centre, Paris, France), a medical centre subsidised by the French national healthcare system (Sécurite Sociale-CNAM) that offers free medical examinations for all affiliated working and retired people and their families. The IPC Centre has carried out approximately 20,000-25,000 examinations per year since 1970 for people living in the Paris area. Between January 
1991 and December 2008, 301,948 participants (193,221 men and 108,727 women), aged 16-95 years (mean \pm SD $44.8 \pm$ 12.0 years for men and $45.1 \pm 14.2$ years for women), underwent a health check at the IPC Centre. All data collected in standard conditions during the health checks-up were used for statistical analysis. Participants with known diabetes $(<9 \%)$ were excluded from the analysis. Data on mortality rates and causes were available for a maximum follow-up of 17 years (mean \pm SD 9.2 \pm 4.7 years). During this period, 5616 deaths were recorded for men (2488 deaths from cancer and 3128 from other causes) and 2032 deaths for women (904 from cancer and 1128 from other causes).

The IPC Centre received authorisation from the French ethics committees (Comité National d'Informatique et des Libertés and Comité Consultatif sur le Traitement de l'Information en matière de Recherche dans le domaine de la Santé) to conduct analyses on all data collected during the health checks. All participants provided informed consent at the time of the examination. Based on national mortality statistics, our cohort presented a lower mortality rate than other French cohorts or the general population. However, the distribution of the different causes of mortality in our cohort was identical to that of the general population in France.

Investigations Blood glucose and other biological variables, including total plasma cholesterol and triacylglycerols, were measured under fasting conditions. Fasting was defined as $12 \mathrm{~h}$ from last meal, or for an appointment at 12:00 hours a coffee or tea and a breakfast rusk without butter or sugar was authorised before 07:00 hours. A blood sample was taken at the beginning of the health check. Measurements were conducted using enzymatic (automat HITACHI 917, Boehringer Mannheim, Basel, Switzerland) and colorimetric methods for albumin dosage, and the ABX Pentra 120 (Horiba, Kyoto, Japan) for haematology. All biological and clinical variables were evaluated on the day of the examination. Supine BP was measured in the right arm three times, from which the mean of the last two measurements was calculated and included in the database. BP was measured by a trained nurse using a manual mercury sphygmomanometer until mid-1998, after which time validated digital electronic BP devices were used (A\&D TM-2541, A\&D Company, Tokyo, Japan). The measurements were conducted after a 10 min rest enabling a resting ECG recording. Height (using a wall-mounted stadiometer) and weight (using calibrated scales) were recorded by a nurse. Heart rate was measured by ECG. Left ventricular hypertrophy was defined according to the Sokolow-Lyon criteria for ECG. Tobacco use, physical activity, personal and family medical history, current medication intake (including glucose-lowering agents and regular aspirin), and alcohol consumption were assessed using a standardised self-administered questionnaire completed during the health check.
Endpoints For each screened participant, vital status was obtained from the Institut National de Statistiques et d'Etudes Economiques (INSEE, Paris, France). Cause of death, obtained from death certificates, was provided by the Institut National de la Santé et de la Recherche Médicale (Inserm) Department of Mortality Studies (Unit SC8). Cause of death was codified according to the ICD-8 (until 1978) or ICD-9 (thereafter; www. who.int/classifications/icd/en/). Data for cause of death were only available up to December 2008. To validate this procedure, a random sample of 364 participants listed as deceased in our database were evaluated in 2012 and the data were compared with those provided by city hall registries for each individual. Discordance was found in only two cases $(0.55 \%)$. Based on the results of this validation, it was considered that we had a complete follow-up of deceased people for the entire study population.

Data analysis Participants with a personal medical history of cancer (4344 women and 2291 men) were excluded from the analysis.

Using blood glucose level quintiles, we performed an analysis of the mortality risk trend according to blood glucose level and a categorical analysis. First, the population was divided into quintiles (Q1-Q5), separately for men and women. Groups were compared using an analysis of variance. Risk of death (all-cause, cancer-related, non-cancer-related) and all types of cancers were compared for each quintile using HR (95\% CI). The first quintile was chosen as the reference group. Excess risk of death was tested through three different models: (1) adjusted for sex and age; (2) adjusted for sex, age and glucose-lowering medication; and (3) full adjustment, including sex, age, glucose-lowering medication, weight, height, BP, heart rate, cholesterol, triacylglycerols, depression score, familial medical history (diabetes, hypertension, myocardial infarction and breast cancer), tobacco use, antihypertensive treatment, ECG abnormalities, socio-professional categories and declared aspirin treatment. Second, blood glucose was considered as a continuous variable. Mortality risk was evaluated for an increase of $0.56 \mathrm{mmol} / 1$ blood glucose with the Cox regression polynomial splines model. A supplementary analysis including lipid-lowering treatment (e.g. statins) was performed. Specific details pertaining to glucoselowering and lipid-lowering treatments were not available in our database. Data are expressed as mean \pm SD. Statistical analyses were performed with SAS software (v8.02) (https:// www.sas.com/fr_fr/home.html).

\section{Results}

The population included 301,948 participants without a personal history of cancer $(193,221$ men, $44.8 \pm 12.0$ years and 108,727 women, $45.1 \pm 14.2$ years). Table 1 summarises the 
Table 1 Baseline blood glucose level (mmol/l) by quintile according to sex

\begin{tabular}{llllll}
\hline & Q1 & Q2 & Q3 & Q4 & Q5 \\
\hline Men & & & & & \\
$\quad n$ & 40,509 & 35,644 & 36,788 & 42,987 & 36,626 \\
$\quad$ Mean (SD) & $4.81(0.24)$ & $5.29(0.08)$ & $5.49(0.08)$ & $5.84(0.13)$ & $6.88(1.61)$ \\
$\quad$ Min. - max. & $2.00-5.06$ & $5.11-5.33$ & $5.39-5.61$ & $5.67-6.06$ & $6.11-32.33$ \\
Women & & & & & \\
$\quad n$ & 22,954 & 21,134 & 21,772 & 19,933 & 22,486 \\
$\quad$ Mean (SD) & $4.55(0.21)$ & $4.95(0.08)$ & $5.22(0.08)$ & $5.52(0.09)$ & $6.32(1.29)$ \\
$\quad$ Min. - max. & $3.06-4.78$ & $4.83-5.06$ & $5.11-5.33$ & $5.39-5.67$ & $5.72-29.33$ \\
\hline
\end{tabular}

Max., maximum; Min., minimum mean \pm SD blood glucose levels for men and women for each quintile, along with minimum and maximum values. The mean \pm SD blood glucose levels in men were: $\mathrm{Q} 1,4.81 \pm 0.24 \mathrm{mmol} /$ l; Q2, $5.29 \pm 0.08 \mathrm{mmol} / \mathrm{l} ; \mathrm{Q} 3,5.49 \pm 0.08 \mathrm{mmol} / \mathrm{l} ; \mathrm{Q} 4,5.84 \pm$ $0.13 \mathrm{mmol} / \mathrm{l}$; and Q5, $6.88 \pm 1.61 \mathrm{mmol} / \mathrm{l}$. Overall, blood glucose levels were lower in women than in men. The blood glucose level that typically defines normoglycaemia was observed in Q3 (5.49 $\pm 0.08 \mathrm{mmol} / 1$ [5.39-5.61] in men and 5.22 $\pm 0.08 \mathrm{mmol} / 1$ [5.11-5.33] in women), and the mean blood glucose level in Q5 was lower than the threshold defining diabetes $(7 \mathrm{mmol} / \mathrm{l})$ in both men and women.

The general characteristics of the population are shown in Table 2. Mean age increased from Q1 to Q5 (39 \pm 12 years to $51 \pm 11$ years, respectively). Glucose-lowering agents (considered as a diagnosis of diabetes in this analysis) were used by $6.06 \%$ of participants, with the lowest proportions in the first three quintiles (3.38-5.44\%) and reaching $8.90 \%$ in Q5. Declared aspirin treatment was similarly distributed at approximately $20 \%$.
Table 3 presents the percentage of deaths attributed to cancer and non-cancer, according to sex, over the median 9 years of follow-up. Mortality rates increased from Q1 to Q5: all-cause, from $1.47 \%$ to $5.54 \%$ in men and from $0.69 \%$ to $3.99 \%$ in women; cancer-related, from $0.59 \%$ to $2.57 \%$ in men and from $0.30 \%$ to $1.69 \%$ in women; non-cancer-related, from $0.88 \%$ to $2.97 \%$ in men and from $0.39 \%$ to $2.30 \%$ in women $(p<0.0001$ for all comparisons for linear trends in men and women). However, it is important to note at this stage that risk of death was significantly increased among participants in Q5.

Table 4 shows the adjusted mortality risk in each blood glucose quintile compared with Q1 (reference group) according to the different models. The relationship between blood glucose and cancer-related death does not appear to be linear, particularly in comparison with all-cause mortality. The HR for allcause mortality went from $1.13(\mathrm{Q} 2)$ to $0.93(\mathrm{Q} 4)$ to $1.16(\mathrm{Q} 5)$, while the HR for cancer-related death went from $1.10(\mathrm{Q} 2)$ to $1.11(\mathrm{Q} 3)$ to $1.04(\mathrm{Q} 4)$ and then rose to $1.36(\mathrm{Q} 5)$. An important result is that the blood glucose level that typical defines
Table 2 Characteristics of the study population by blood glucose level quintile

\begin{tabular}{llllll}
\hline & Q1 & Q2 & Q3 & Q4 & Q5 \\
\hline Age (years) & $39(12)$ & $43(13)$ & $45(13)$ & $47(12)$ & $51(11)$ \\
BMI $\left(\mathrm{kg} / \mathrm{m}^{2}\right)$ & $23.8(3.8)$ & $24.3(3.8)$ & $24.6(3.8)$ & $25.1(3.8)$ & $26.1(4.3)$ \\
BP $(\mathrm{mmHg})$ & $124(16)$ & $126(16)$ & $128(16)$ & $131(16)$ & $135(18)$ \\
Heart rate $(\mathrm{bpm})$ & $62(10)$ & $64(10)$ & $65(11)$ & $66(11)$ & $70(12)$ \\
$\gamma$-glutamyltransferase (U/l) & $25.8(34.4)$ & $26.6(34.2)$ & $27.4(36.1)$ & $30.0(44.4)$ & $37.0(63.3)$ \\
Cholesterol (mmol/l) & $5.22(1.06)$ & $5.46(1.07)$ & $5.60(1.08)$ & $5.76(1.1)$ & $5.98(1.14)$ \\
Cholesterol treatment & 2.55 & 3.39 & 4.23 & 4.92 & 7.47 \\
Leucocytes $\left(10^{9} / \mathrm{l}\right)$ & $6.62(2.0)$ & $6.68(1.9)$ & $6.72(1.9)$ & $6.78(1.8)$ & $6.89(1.9)$ \\
Depression score & $1.79(2.75)$ & $1.72(2.68)$ & $1.70(2.64)$ & $1.68(2.60)$ & $1.93(2.77)$ \\
Current tobacco use & 15.7 & 17.1 & 19.5 & 23.6 & 24.2 \\
Glucose-lowering treatment & 5.44 & 3.38 & 4.83 & 7.49 & 8.90 \\
Aspirin & 21.26 & 18.97 & 19.45 & 20.80 & 19.52 \\
Family history of breast cancer & 6.52 & 6.98 & 7.25 & 7.10 & 6.98 \\
Family history of intestinal cancer & 3.54 & 3.99 & 4.36 & 4.77 & 5.42 \\
Physical activity & 22.74 & 19.77 & 19.81 & 20.64 & 17.0 \\
\hline
\end{tabular}

Data are expressed as mean (SD) or percentage 
Table 3 All-cause, cancer- and non-cancer-related mortality rates, according to sex, over the median 9 year follow-up

\begin{tabular}{|c|c|c|c|c|c|}
\hline & Q1 & Q2 & Q3 & Q4 & Q5 \\
\hline \multicolumn{6}{|l|}{ All-cause } \\
\hline Men & 1.47 (592) & 1.95 (696) & 2.54 (933) & $3.21(1374)$ & $5.54(2021)$ \\
\hline Women & 0.69 (158) & 0.97 (204) & 1.59 (346) & $2.15(429)$ & $3.99(895)$ \\
\hline \multicolumn{6}{|l|}{ Cancer } \\
\hline Men & 0.59 (238) & $0.76(271)$ & $1.16(426)$ & $1.44(615)$ & 2.57 (938) \\
\hline Women & $0.30(68)$ & $0.48(101)$ & $0.73(158)$ & 0.99 (198) & 1.69 (379) \\
\hline \multicolumn{6}{|l|}{ Non-cancer } \\
\hline Men & $0.88(354)$ & $1.19(425)$ & $1.38(507)$ & 1.77 (759) & $2.97(1083)$ \\
\hline Women & $0.39(90)$ & 0.49 (103) & $0.86(188)$ & $1.16(231)$ & $2.30(516)$ \\
\hline
\end{tabular}

Data are expressed as \% (n)

$P<0.0001$ for trend for all comparisons for men and women normoglycaemia is not associated with an excess risk of cancer-related death mortality.

In the Q5, an increased risk of all-cause and cancer-related death was observed after adjustment for age and sex. This excess risk remained significant after adjustment for age, sex and glucose-lowering treatment (model 2). After full adjustment (model 3), the excess risk of all-cause death was no longer present. In contrast, a significantly lower mortality rate was noted in Q4 compared with Q1. For cancer-related death, after full adjustment, the excess risk associated with Q5 reached $17 \%$ (HR [95\% CI] 1.17 [1.03, 1.34]), corresponding to population attributable proportion of cancer of $3.22 \%$. Additional analyses including lipid-lowering treatment did not modify this result (data not shown). For non-cancer- related death, analysis by quintile did not reveal any excess risk of death associated with blood glucose level.

Considering blood glucose as a continuous variable, the risk of death (HR [95\% CI]) associated with an increase of $0.56 \mathrm{mmol} / \mathrm{l}$ of blood glucose was $1.04(1.02,1.06)$ for cancer, $1.04(1.03,1.06)$ for non-cancer and $1.04(1.03,1.05)$ for allcause. The relationship with blood glucose remained significant after the introduction of a quadratic term (blood glucose squared) in the model for cancer-related deaths but disappeared for non-cancer-related deaths.

Table 5 shows the risk of cancer-related death according to normoglycaemia or hyperglycaemia (blood glucose $\geq 7$ $\mathrm{mmol} / \mathrm{l})$ status and the use of glucose-lowering medication. Only the group presenting with high blood glucose levels
Table 4 Nine year mortality risk according to blood glucose level quintile

\begin{tabular}{|c|c|c|c|c|c|}
\hline & $\mathrm{Q}^{\mathrm{a}}$ & Q2 vs Q1 & Q3 vs Q1 & Q4 vs Q1 & Q5 vs Q1 \\
\hline \multicolumn{6}{|l|}{ All-cause } \\
\hline Model $1^{\mathrm{b}}$ & 1 & $1.13(1.03,1.24)$ & $1.00(0.92,1.08)$ & $0.93(0.86,1.00)$ & $1.16(1.07,1.24)$ \\
\hline Model $2^{\mathrm{c}}$ & 1 & $1.13(1.03,1.24)$ & $0.99(0.92,1.08)$ & $0.93(0.86,1.00)$ & $1.14(1.07,1.23)$ \\
\hline Model $3^{\mathrm{d}}$ & 1 & $1.08(0.98,1.19)$ & $0.98(0.90,1.07)$ & $0.90(0.83,0.98)$ & $0.98(0.89,1.06)$ \\
\hline \multicolumn{6}{|l|}{ Cancer } \\
\hline Model $1^{\mathrm{b}}$ & 1 & $1.10(0.94,1.28)$ & $1.11(0.97,1.26)$ & $1.04(0.92,1.18)$ & $1.36(1.21,1.54)$ \\
\hline Model $2^{\mathrm{c}}$ & 1 & $1.10(0.95,1.28)$ & $1.11(0.97,1.26)$ & $1.04(0.92,1.18)$ & $1.36(1.21,1.53)$ \\
\hline Model $3^{\mathrm{d}}$ & 1 & $1.04(0.881 .23)$ & $1.07(0.93,1.22)$ & $0.99(0.87,1.13)$ & $1.17(1.03,1.34)$ \\
\hline \multicolumn{6}{|l|}{ Non-cancer } \\
\hline Model $1^{\mathrm{b}}$ & 1 & $1.07(0.95,1.22)$ & $0.95(0.85,1.06)$ & $0.91(0.82,1.02)$ & $1.10(1.00,1.22)$ \\
\hline Model $2^{\mathrm{c}}$ & 1 & $1.07(0.94,1.22)$ & $0.95(0.85,1.06)$ & $0.91(0.82,1.01)$ & $1.08(0.97,1.19)$ \\
\hline Model $3^{d}$ & 1 & $1.02(0.89,1.17)$ & $0.94(0.83,1.06)$ & $0.89(0.79,1.00)$ & $0.91(0.81,1.01)$ \\
\hline
\end{tabular}

Data are expressed as HR $(95 \% \mathrm{CI})$

${ }^{a}$ Reference group

${ }^{\mathrm{b}}$ Model adjusted for age and sex

${ }^{\mathrm{c}}$ Model adjusted for age, sex and glucose-lowering treatment

${ }^{\mathrm{d}}$ Model adjusted for age, sex, cholesterol, weight, BP, triacylglycerols, heart rate, depression, family medical history (diabetes, hypertension, myocardial infarction, breast cancer and intestinal cancer), tobacco use, physical activity, leucocyte levels, glucose-lowering treatment, ECG abnormalities, socio-professional categories, aspirin use 
Table 5 Risk of cancer-related death according to blood glucose status and glucose-lowering treatment use

\begin{tabular}{lllll}
\hline & NG-NTT $^{\mathrm{a}}$ & HG-NTT & NG-TT & HG-TT \\
\hline Adjusted for age and sex & 1 & $1.52(1.33,1.72)^{* * * *}$ & $1.04(0.70,1.56)$ & $1.16(0.85,1.59)$ \\
Full adjustment $^{\mathrm{b}}$ & 1 & $1.26(1.10,1.45)^{* *}$ & $1.06(0.69,1.61)$ & $1.03(0.74,1.43)$ \\
\hline
\end{tabular}

Data are expressed as HR $(95 \% \mathrm{CI})$

${ }^{a}$ Reference group

${ }^{\mathrm{b}}$ Model adjusted for age, sex, cholesterol, weight, BP, triacylglycerols, heart rate, depression, family history (diabetes, hypertension, myocardial infarction, breast cancer and intestinal cancer), tobacco use, physical activity, leucocyte levels, glucose-lowering treatment, ECG abnormalities, socio-professional category and aspirin use

$* * p<0.01, * * * p<0.001 \mathrm{vs}$ the reference group (NG-NTT)

NG-NTT, normoglycaemia and no glucose-lowering treatment; HG-NTT: hyperglycaemia (>7 mmol/1) and no glucose-lowering treatment; NG-TT, normoglycaemia with glucose-lowering treatment; HG-TT, hyperglycaemia (>7 mmol/l) with glucose-lowering treatment without treatment was exposed to a significantly increased risk of cancer-related death.

After full adjustment, participants with a blood glucose level $\geq 7 \mathrm{mmol} / 1$ but who were not receiving glucoselowering treatment, considered as incident diabetes, had an excess risk of cancer-related death (HR [95\% CI] 1.26 [1.10, 1.45]). This risk was similar for non-cancer-related death (HR $[95 \% \mathrm{CI}] 1.66[1.49,1.86])$. This excess risk for cancerrelated death became non-significant when participants were treated for diabetes irrespective of their glucose control. However, participants receiving glucose-lowering medication, considered as having 'known diabetes', presented an excess of risk of non-cancer-related death (HR [95\% CI] $1.45[1.10,1.92]$ for normoglycaemia and $1.60[1.29,1.99]$ for hyperglycaemia). These results suggest that incidence of diabetes is associated with cancer- and non-cancer-related death, and that glucose-lowering medication reduces the risk of cancer-related but not non-cancer-related death.

These results have significant implications for additional benefits of glucose-lowering medications on reducing cancerrelated deaths. Although detailed information on prescription of glucose-lowering medications was not available, such a long-term effect of treatment is worth emphasising.

The relationship between blood glucose and cancer-related death differed according to the type of cancer (Table 6). For gastrointestinal cancer and leukaemia, an excess risk of death was observed in Q5, which remained significant after full adjustment (50\% and 78\%, respectively). For lung and prostate cancers, the significantly higher risk of death associated with Q5 disappeared after adjustment for confounders. For kidney and breast cancers, there were no associations between blood glucose level and mortality rates. For other cancers, the risk of death was significantly lower, regardless of the model used, suggesting a low incidence of cancer when blood glucose is within the normal range. For other cancers, the risk of death was significantly lower, regardless of the model used, when blood glucose is within the normal range, compared with higher blood glucose, and a low incidence of cancer was observed.
Tobacco-related cancers were grouped together (lung, mouth and throat, stomach, and bladder) and their relationship was analysed according to tobacco use. The relationship for cancer-related death and hyperglycaemia was not statistically significantly different between smokers and non-smokers with tobacco-related cancers (HR [95\% CI] 1.19 [0.88, 1.60] and $1.45[1.08,1.95]$, respectively,). A non-linear trend between cancer-related death and blood glucose level was also observed for specific cancers, confirming a non-coincidental result for the study.

\section{Discussion}

The main finding of this study is an increased mortality rate associated with higher blood glucose levels in a large primary care population presenting a low rate of diabetes. Hyperglycaemia had a negative impact regardless of the cause of death in our cohort. For the purposes of this analysis, all those treated with glucose-lowering medication were considered to have diabetes. As a result of our inclusion criteria, which required only one measurement of fasting blood glucose, we were unable to identify individuals with non-treated diabetes. Only a small proportion of the study population had diabetes (Table 2; 3.38-8.90\%) and use of glucose-lowering treatment was adjusted for in the statistical analysis.

The main finding of the present study is that, after adjustment for confounding factors, mortality rates related to cancer increased in both men and women who presented with high levels of fasting blood glucose. The mean blood glucose level on Q5 reached $6.88 \mathrm{mmol} / \mathrm{l}$ for 36,626 male participants and $6.52 \mathrm{mmol} / \mathrm{l}$ for 22,486 female participants, which represents only moderate increases relative to the normal range (i.e. below the threshold defining diabetes). The increase in risk of cancerrelated death was approximately $18 \%$ after full adjustment. In contrast, the HR for non-cancer-related and all-cause deaths for Q5 were comparable with Q1 (reference group). This study specifically focused on blood glucose, and not diabetes, in the main analysis, thereby confirming a deleterious relationship 
Table 6 Mortality risk for various types of cancer according to blood glucose quintile

\begin{tabular}{|c|c|c|c|c|c|}
\hline Models for adjustment & $\mathrm{Q} 1^{\mathrm{a}}$ & Q2 vs Q1 & Q3 vs Q1 & Q4 vs Q1 & Q5 vs Q1 \\
\hline \multicolumn{6}{|l|}{ Lung cancer $(n=818)$} \\
\hline Model $1^{\mathrm{b}}$ & 1 & $1.23(0.91,1.66)$ & $1.25(0.96,1.62)$ & $1.04(0.80,1.34)$ & $1.42(1.11,1.81)$ \\
\hline Model 2 & 1 & $1.24(0.92,1.67)$ & $1.25(0.96,1.62)$ & $1.04(0.81,1.34)$ & $1.43(1.12,1.82)$ \\
\hline Model $3^{\text {d }}$ & 1 & $1.05(0.77,1.45)$ & $1.17(0.89,1.55)$ & $1.01(0.77,1.31)$ & $1.22(0.94,1.60)$ \\
\hline \multicolumn{6}{|c|}{ Gastrointestinal cancer $\mathrm{f}(n=378)$} \\
\hline Model $1^{\mathrm{b}}$ & 1 & $1.44(0.91,2.28)$ & $1.17(0.77,1.78)$ & $1.22(0.82,1.82)$ & $1.71(1.18,2.49)$ \\
\hline Model $2^{\mathrm{c}}$ & 1 & $1.45(0.91,2.29)$ & $1.17(0.77,1.80)$ & $1.22(0.82,1.81)$ & $1.75(1.20,2.54)$ \\
\hline Model $3^{\mathrm{d}}$ & 1 & $1.58(0.97,2.61)$ & $1.14(0.72,1.79)$ & $1.19(0.78,1.83)$ & $1.50(1.00,2.27)$ \\
\hline \multicolumn{6}{|l|}{ Kidney cancer $(n=164)$} \\
\hline Model $1^{\mathrm{b}}$ & 1 & $0.52(0.23,1.18)$ & $1.00(0.57,1.79)$ & $1.00(0.58,1.71)$ & $1.10(0.66,1.84)$ \\
\hline Model $2^{\mathrm{c}}$ & 1 & $0.52(0.23,1.19)$ & $1.01(0.57,1.79)$ & $1.00(0.59,1.72)$ & $1.10(0.66,1.85)$ \\
\hline Model $3^{\mathrm{d}}$ & 1 & $0.54(0.23,1.26)$ & $1.04(0.57,1.90)$ & $1.03(0.58,1.81)$ & $0.95(0.54,1.65)$ \\
\hline \multicolumn{6}{|l|}{ Leukaemia $(n=201)$} \\
\hline Model $1^{\mathrm{b}}$ & 1 & $0.84(0.39,1.80)$ & $1.56(0.88,2.77)$ & $1.54(0.89,2.67)$ & $1.76(1.03,3.01)$ \\
\hline Model $2^{\mathrm{c}}$ & 1 & $0.85(0.40,1.81)$ & $1.55(0.87,2.75)$ & $1.55(0.90,2.68)$ & $1.76(1.03,3.01)$ \\
\hline Model $3^{\mathrm{d}}$ & 1 & $0.88(0.41,1.83)$ & $1.46(0.82,2.61)$ & $1.31(0.74,2.30)$ & $1.78(1.00,3.15)$ \\
\hline \multicolumn{6}{|l|}{ Breast cancer $(n=148)$} \\
\hline Model $1^{\mathrm{b}}$ & 1 & $0.88(0.42,1.45)$ & $0.81(0.44,1.50)$ & $1.14(0.65,2.00)$ & $1.06(0.61,1.84)$ \\
\hline Model $2^{\mathrm{c}}$ & 1 & $0.88(0.42,1.87)$ & $0.81(0.44,1.50)$ & $1.14(0.65,2.00)$ & $1.07(0.61,1.85)$ \\
\hline Model $3^{\mathrm{d}}$ & 1 & $0.89(0.40,1.97)$ & $0.78(0.41,1.52)$ & $1.10(0.60,2.01)$ & $0.97(0.52,1.78)$ \\
\hline \multicolumn{6}{|c|}{ Prostate cancer $(n=217)$} \\
\hline Model $1^{\mathrm{b}}$ & 1 & $1.52(0.83,2.78)$ & $1.06(0.59,1.88)$ & $0.92(0.53,1.59)$ & $1.79(1.09,2.93)$ \\
\hline Model $2^{\mathrm{c}}$ & & $1.54(0.84,2.81)$ & $1.06(060,1.89)$ & $0.91(0.53,1.58)$ & $1.80(1.09,2.95)$ \\
\hline Model $3^{\text {d }}$ & 1 & $1.74(0.92,3.29)$ & $1.03(0.55,1.88)$ & $0.87(0.49,1.56)$ & $1.53(0.90,2.63)$ \\
\hline \multicolumn{6}{|c|}{ Other cancers $(n=1246)$} \\
\hline Model $1^{\mathrm{b}}$ & 1 & $1.18(0.99,1.41)$ & $0.95(0.81,1.12)$ & $0.84(0.72,0.98)$ & $1.06(0.92,1.23)$ \\
\hline Model $2^{\mathrm{c}}$ & & $1.18(0.99,1.41)$ & $0.95(0.81,1.11)$ & $0.84(0.72,0.96)$ & $1.05(0.90,1.21)$ \\
\hline Model $3^{\mathrm{d}}$ & 1 & $1.15(0.95,1.40)$ & $0.97(0.82,1.16)$ & $0.83(0.70,0.98)$ & $0.94(0.80,1.11)$ \\
\hline
\end{tabular}

Data are expressed as HR $(95 \% \mathrm{CI})$

${ }^{a}$ Reference group

${ }^{\mathrm{b}}$ Model adjusted for age and sex

${ }^{\mathrm{c}}$ Model adjusted for age, sex and glucose-lowering treatment

${ }^{\mathrm{d}}$ Model adjusted for age, sex, cholesterol, weight, BP, triacylglycerols, heart rate, depression, family medical history (diabetes, hypertension, myocardial infarction, breast cancer and intestinal cancer), tobacco use, physical activity, leucocyte levels, glucose-lowering treatment, ECG abnormalities, socio-professional categories, aspirin use

between altered glucose metabolism and risk of cancer; therefore, the results carry greater significance than a confirmatory result linking diabetes and cancer. Two recent studies have also reported a similar relationship between blood glucose level and cancer risk. In the Hisayama study conducted in a Japanese general population, the risk of cancer-related death was almost doubled when fasting plasma glucose levels were $>5.6 \mathrm{mmol} / \mathrm{l}$ [5]. While in a subanalysis of the Apolipoprotein MOrtality RISk (AMORIS) study incorporating cancer data from the Swedish National Cancer Register, a positive trend (+8\%) was observed between mean glucose level and overall cancer risk [6]. The findings presented here add a new dimension to the above. First, the range of blood glucose levels was broad and not limited to one arbitrary threshold as in the Hisayama study. Second, we were able to compare cancer mortality rates with non-cancer mortality rates in contrast to the AMORIS study. Third, our population had a much larger sample size than the other studies. Although we were unable to obtain detailed data regarding glucose-lowering treatments, we nevertheless compared participants grouped according to blood glucose level (normo or hyperglycaemia) and the presence or absence of treatment (Table 5). Overall, our results confirm a significant increase in cancer-related deaths associated with hyperglycaemia, although only when observed in the absence of glucose-lowering treatment. It would appear that glucoselowering treatment stabilises the risk of cancer-related death 
to levels similar to those observed in participants with normoglycaemia, i.e. the group with normal blood glucose without treatment. However, we defined diabetes using a threshold of $>7 \mathrm{mmol} / \mathrm{l}$ blood glucose. Furthermore, blood glucose was only measured once and glucose-lowering treatment use was declared by the participant through a self-administered questionnaire. Although a clear association has been demonstrated between metformin use and reduced risk of death in type 2 diabetes, we have shown here an additional benefit of glucose-lowering treatment: a reduce risk of cancer even with residual hyperglycaemia during treatment (Table 5). A clinical trial in individuals with type 2 diabetes where a large initial $\mathrm{HbA}_{1 \mathrm{c}}$ reduction and achievement of low $\mathrm{HbA}_{1 \mathrm{c}}$ levels within 6 months of metformin treatment initiation are associated with a lower risk of cardiovascular events and death [13].

The originality of the present study is the analysis of mortality rates related to a range of specific cancers according to blood glucose level. A number of studies have demonstrated a relationship, albeit weak, between blood glucose level and breast cancer or metastasis [14-16]. This relationship was not confirmed herein; although our endpoint was death and not cancer incidence, which was shown to be increased up to $63 \%$ in the ORDET study [14]. Moreover, the association between blood glucose and death related to lung cancer retrospectively found in a small population [17] was not observed. Although a significant association with gastrointestinal cancers was observed herein, we were unable to specifically distinguish pancreatic cancer in our database. Indeed, there are conflicting reports regarding blood glucose and pancreatic cancer. The latter has been found to be associated with hyperglycaemia [18], but also associated or solely with circulating markers of peripheral insulin resistance but not hyperglycaemia [19]. Finally, we observed, in both analyses of overall cancer-related death and in a majority of deaths related to specific cancers, a similar non-linear trend between blood glucose quintile and mortality rate after adjustment for age and after full adjustment (Table 4), while the raw data (Table 3) suggested a linear relationship. Consequently our data indicate two major findings: (1) within the normal range, in both sexes, blood glucose is not associated with an increased risk of cancer; and (2) the highest blood glucose level is significantly associated with cancer-related but not noncancer-related death.

A relationship between BMI and specific cancers has been well established in a recent population-based study [20], in which each $5 \mathrm{~kg} / \mathrm{m}^{2}$ increase was associated with particular cancer types. In our population, BMI increased slightly with blood glucose quintile, from $23.8 \mathrm{~kg} / \mathrm{m}^{2}$ to $26.1 \mathrm{~kg} / \mathrm{m}^{2}$ (Table 2); the final analysis was therefore adjusted for weight to take this confounding factor into account.

Of note, tobacco use cancelled the relationship between tobacco-related cancers and blood glucose because of its overwhelming deleterious action. Finally, our results show that hyperglycaemia was associated with cancer-related death but not with causes of death usually associated with diabetes, such as cardiovascular disease. Given that the present study was epidemiological as opposed to mechanistic in design, it is not possible to draw conclusions on specific causality or to provide a definite explanatory hypothesis. However, the hypothesis suggesting a role of common risk factors or general inflammation remains appropriate in this instance [21,22].

A protective effect of aspirin has been suggested against metastasis and cancer-related death, specifically for colorectal cancer as shown in a meta-analysis of large-scale studies. In the present study, we took into consideration the presence of treatment with aspirin as reported by approximately one-fifth of the participants in each blood glucose quintile. Again, a relationship between hyperglycaemia and cancer-related death was maintained after adjusting for the use of aspirin. This point further strengthens our findings and suggests that aspirin treatment did not play a favourable role in this relationship.

Limitations of the study There are a number of limitations associated with this study that should be acknowledged. First, blood glucose was only measured once and was not confirmed in subsequent measurements. However, blood sample collection was performed in fasting and standardised conditions, and the positive relationship between blood glucose level and death would not be impacted by this single measurement. Furthermore, the analysis using quintiles should limit any effect on the observed results.

Details regarding glucose-lowering treatment were not available, such that only presence or absence of medication use was known. Hence, no conclusions can be drawn regarding a potential impact of these drugs. Similarly, specific details regarding lipid-lowering treatment (i.e. statins) were not available.

Second, we only addressed mortality rates in this analysis and not the incidence of cancer. Thus, the conclusions of this study are not generalisable to cancer incidence but rather specific to cancer-related death. Furthermore, our follow-up for causes of death in this specific cohort ended in 2008, which is a clear limitation for participants aged $45 \pm 12$ years at the time of inclusion.

Finally, $\mathrm{HbA}_{1 \mathrm{c}}$ was not available in our database and we did not assess the $2 \mathrm{~h}$ OGTT. A recent study by Goto et al showed that the highest levels of $\mathrm{HbA}_{1 \mathrm{c}}$ within the nondiabetic range were associated with cancer incidence [23]. The large Diabetes Epidemiology: Collaborative analysis of Diagnostic criteria in Europe (DECODE) study showed that in individuals without diabetes, cancer-related mortality rates were similarly increased for fasting and $2 \mathrm{~h}$ OGTT plasma glucose [24].

In conclusion, the large-scale study presented here shows that, compared with non-cancer-related death, fasting hyperglycaemia was significantly associated with cancer- 
related death after adjusting for confounding factors, in particular treatments with aspirin or glucose-lowering medications.

Although not mechanistic in design, the findings of this study putatively suggest that common factors or inflammation associated with glucose metabolism may play a deleterious role in the evolution of cancer, if not its occurrence.

Acknowledgements We thank the Caisse Nationale d'Assurance Maladie des Travailleurs Salariés (CNAM-TS, France) and the Caisse Primaire d'Assurance Maladie de Paris (CPAM-P, France) for their help in making this study possible.

Data availability All data are available from the IPC Centre database and a partnership agreement must be signed in order to use it.

Funding This research received no specific grant from any funding agency in the public, commercial or not-for-profit sectors.

Duality of interest The authors declare that there is no duality of interest associated with this manuscript.

Contribution statement JMS, BP and FT made substantial contributions to conception, design, interpretation of the results and drafting of the manuscript. FT performed the statistical analysis. SC, OH, CL, TS, ND contributed to the conception and design of the study, interpretation of the data and critically revised the manuscript for important intellectual content. All authors approved the final manuscript to be published. BP is guarantor and is therefore responsible for the integrity of the work as a whole.

\section{References}

1. Giovannucci E, Harlan DM, Archer MC et al (2010) Diabetes and cancer: a consensus report. CA Cancer J Clin 60:207-221

2. Noto H, Tsujimoto T, Sasazuki T, Noda M (2011) Significantly increased risk of cancer in patients with diabetes mellitus: a systematic review and meta-analysis. Endocr Pract 17:616-628

3. Chen Y, Wu F, Saito E et al (2017) Association between type 2 diabetes and risk of cancer mortality: a pooled analysis of over 771,000 individuals in the Asia Cohort Consortium. Diabetologia 60:1022-1032

4. Luo J, Lin HC, He K, Hendryx M (2014) Diabetes and prognosis in older persons with colorectal cancer. Br J Cancer 111:1847-1854

5. Hirakawa Y, Ninomiya T, Mukai N et al (2012) Association between glucose tolerance level and cancer death in a general Japanese population: the Hisayama Study. Am J Epidemiol 176:856-864

6. Wulaningsih W, Holmberg L, Garmo H et al (2013) Serum glucose and fructosamine in relation to risk of cancer. PLoS One 8:e54944

7. DeCensi A, Puntoni M, Goodwin P et al (2010) Metformin and cancer risk in diabetic patients: a systematic review and metaanalysis. Cancer Prev Res (Phila) 3:1451-1461

8. Colmers IN, Bowker SL, Tjosvold LA, Johnson JA (2012) Insulin use and cancer risk in patients with type 2 diabetes: a systematic review and meta-analysis of observational studies. Diabete Metab 38:485-506

9. Bordeleau L, Yakubovich N, Dagenais GR et al (2014) The association of basal insulin glargine and/or n-3 fatty acids with incident cancers in patients with dysglycemia. Diabetes Care 37:1360-1366

10. Rothwell PM, Price JF, Fowkes FGR et al (2012) Short-term effects of daily aspirin on cancer incidence, mortality, and non-vascular death: analysis of the time course of risks and benefits in 51 randomised controlled trials. Lancet 379:1602-1612

11. Algra AM, Rothwell PM (2012) Effects of regular aspirin on longterm cancer incidence and metastasis: a systematic comparison of evidence from observational studies versus randomised trials. Lancet Oncol 13:518-527

12. US Preventive Services Task Force (2016) Aspirin use to prevent cardiovascular disease and colorectal cancer: preventive medication. Available from www.uspreventiveservicestaskforce.org/Page/ Document/UpdateSummaryFinal/aspirin-to-preventcardiovascular-disease-and-cancer?ds=1\&s=aspirin $\% 20$ cancer Assessed 23 Mar 2017

13. Svensson E, Baggesen LM, Johnsen SP et al (2017) Early glycemic control and magnitude of $\mathrm{HbA}(1 \mathrm{c})$ reduction predict cardiovascular events and mortality: population-based cohort study of 24,752 metformin initiators. Diabetes Care 40:800-807

14. Sieri S, Muti P, Claudia A et al (2012) Prospective study on the role of glucose metabolism in breast cancer occurrence. Int $\mathrm{J}$ Cancer 130:921-929

15. Contiero P, Berrino F, Tagliabue G et al (2013) Fasting blood glucose and long-term prognosis of non-metastatic breast cancer: a cohort study. Breast Cancer Res Treat 138:951-959

16. Boyle P, Koechlin A, Pizot C et al (2013) Blood glucose concentrations and breast cancer risk in women without diabetes: a metaanalysis. Eur J Nutr 52:1533-1540

17. Luo J, Chen YJ, Chang LJ (2012) Fasting blood glucose level and prognosis in non-small cell lung cancer (NSCLC) patients. Lung Cancer 76:242-247

18. Liao WC, Tu YK, Wu MS, Lin JT, Wang HP, Chien KL (2015) Blood glucose concentration and risk of pancreatic cancer: systematic review and dose-response meta-analysis. BMJ 349:g7371

19. Wolpin BM, Bao Y, Rong Qian Z et al (2013) Hyperglycemia, insulin resistance, impaired pancreatic $\beta$-cell function, and risk of pancreatic cancer. J Natl Cancer Inst 105:1027-1035

20. Bhaskaran K, Douglas I, Forbes H, Dos Santos SI, Leon DA, Smeeth L (2014) Body-mass index and risk of 22 specific cancers: a population-based cohort study of 5.24 million UK adults. Lancet 384:755-765

21. García-Jiménez C, Gutiérrez-Salmerón M, Chocarro-Calvo A, García-Martinez JM, Castaño A, De la Vieja A (2016) From obesity to diabetes and cancer: epidemiological links and role of therapies. Br J Cancer 114:716-722

22. Klil-Drori AJ, Azoulay L, Pollak MN (2017) Cancer, obesity, diabetes, and antidiabetic drugs: is the fog clearing? Nat Rev Clin Oncol 14:85-99

23. Goto A, Noda M, Sawada N et al (2016) High hemoglobin A1c levels within the non-diabetic range are associated with the risk of all cancers. Int J Cancer 138:1741-1753

24. Zhou X, Qiao Q, Zethelius B et al (2010) Diabetes, prediabetes and cancer mortality. Diabetologia 53:1867-1876 